\title{
Preparation of gem-difluorinated retrohydroxamic-fosmidomycin
}

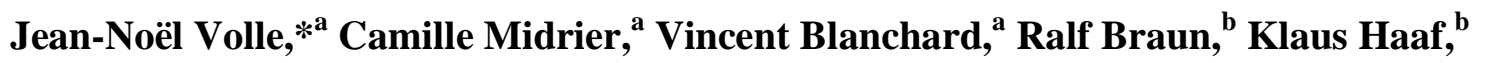 \\ Lothar Willms, ${ }^{b}$ Jean-Luc Pirat ${ }^{a}$, and David Virieux*a \\ ${ }^{a}$ Institut Charles Gerhardt - UMR5253 - AM2N - ENSCM, 8, Rue de l'Ecole Normale, F34296 \\ Montpellier Cedex 5 - France \\ ${ }^{b}$ Bayer CropScience AG, Chemistry Frankfurt, G 836, Industriepark Höchst, 65926 Frankfurt \\ am Main - Germany \\ E-mail: $\underline{\text { Jean-noel.volle@enscm.fr, david.virieux@enscm.fr }}$
}

\begin{abstract}
Dedicated to Professor Manfred Schlosser in honor of his scientific achievements
\end{abstract}
DOI: $\underline{\text { http://dx.doi.org/10.3998/ark.5550190.p008.846 }}$

\begin{abstract}
From several decades, some organophosphorus compounds specifically designed to alter biological systems were introduced on market as agrochemicals (ie glyphosate and glufosinate as herbicides). Nevertheless, it becomes necessary to find new compounds in order to counter plant resistances already observed with glyphosate. Fosmidomicyn and its $\mathrm{N}$-acetyl analogues FR900098 were perceived as starting points for elaboration of new herbicide candidates, targeting the second enzyme of the non-mevalonate pathway in plants, the 1-deoxy-D-xylulose 5phosphate reductoisomerase (DOXP reductoisomerase or DXR). It is expected that the enhancement of bioactivity compared to the parent compounds, might be reached by insertion of two fluorine atoms close to the phosphonate function. Indeed, the presence of both fluorine atoms could improve the lipophilicity, affect the $\mathrm{pKa}$ of the phosphonic acid function and then induce better activities. Herein, the synthesis of gem-difluorinated analogues of retrohydroxamic fosmidomycin and FR-900098-ester is reported using a radical addition mediated by a cobaloxime complex.
\end{abstract}

Keywords: Fosmidomycin, phosphonate, hydroxamic acid, 1-deoxy-D-xylulose 5-phosphate reductoisomerase, DOXP reductoisomerase, DXR

\section{Introduction}

The world population is increasing and different estimations from United Nations planned for 2050 a population ranging from 8 to 10.5 billion people. In order to supply sufficient food, the 
crop productivity has to urgently increase, and elaboration of powerful and ecocompatible new herbicides in absence of mammalian toxicity appears therefore a crucial issue.

The mevalonate-independent pathway of isoprenoid biosynthesis is widely found in many microorganisms ${ }^{1-4}$ as well as in higher plants, ${ }^{5,6}$ but it is missing in human, which uses mevalonate pathway for isoprenoid biosynthesis. The unique property of 1-deoxy-D-xylulose 5phosphate reductoisomerase (DXR), the second enzyme of the DOXP pathway, can therefore be considered as a remarkable and safe target for the discovery of new herbicides. The fosmidomycin 1a isolated from Streptomyces lavendulae in the seventies, has been referenced as an inhibitor of the DXR enzyme, ${ }^{5,7}$ as well as $N$-acetyl homologue FR900098 $\mathbf{1 b},{ }^{8,9}$ fosmidomycin and FR900098 retrohydroxamic acids $\mathbf{1 c}^{10} \mathbf{d}^{10,11}$ and phosphate analogues, namely fosfoxacin $1 \mathbf{e}^{12}$ or acetyl analogue $\mathbf{1 f}^{12}$ (Figure 1). Fosmidomycin $\mathbf{1 a}$ has been positively evaluated for the treatment of uncomplicated falciparum malaria in combination with clindamycin, ${ }^{13-15}$ as well as for its herbicide activities, by use alone in combination with commercial triazine or urea herbicides. ${ }^{16}$ Nevertheless, to date and despite these promising results, fosmidomycin has not been reached on the market neither as antimalarial drug nor as herbicide agent.

In the case of the phosphate derivative $\mathbf{1 e}$ and preferentially 1f, their respective activity clearly revealed stronger than the fosmidomycin on Synechocystis DXR. ${ }^{12}$ These results could be directly attributed to the phosphate function. However, the intrinsic stability of phosphate group in biological media was questionable and clearly constituted its main drawback for a possible herbicide development. From a bioisostere approach, Van Calenbergh, ${ }^{17}$ replaced oxygen atom by a monofluoromethylene group (CHF) and thus, two monofluorinated to FR900098 and to retrohydroxamic-FR900098 analogues were prepared. Both structures displayed that they were, in vitro, more potent than fosmidomycin and FR900098 towards P. Falciparum strain K1, and the antimalarial activity in an in vivo mouse model exceeded those of $\mathbf{1 b} .^{17}$

By comparison to monofluoromethylene group, the difluoromethylene group $\left(\mathrm{CF}_{2}\right)$ exhibits also steric and electronic properties close to oxygen. ${ }^{18-21}$ As a consequence of this equivalence, the $\mathrm{pKa}$ values of the difluorophosphonate are relatively similar to the phosphate, ${ }^{19-21}$ and it might be considered as equivalent to the acidic character of phosphoric group of natural substrate 2. Moreover, the replacement of phosphate bond $\left(\mathrm{HO}_{2} \mathrm{P}(\mathrm{O}) \mathrm{O}-\mathrm{R}\right)$ by a phosphonate linkage $\left(\mathrm{HO}_{2} \mathrm{P}(\mathrm{O}) \mathrm{CF}_{2}-\mathrm{R}\right)$ should increase chemical stability towards phosphatase, favouring a better bioavailability. ${ }^{22}$ Finally, the DXR inhibitor incorporating difluoromethylene group, could have a higher lipophilic character, ${ }^{23}$ allowing potentially a better integration in plants. Therefore, it became obvious that an $\alpha, \alpha^{\prime}$-difluorophosphonate group should advantageously replace the phosphate group of 1f, and preparation of compounds $\mathbf{3}$ and 4, analogues to FR-900098 $\mathbf{1 b}$ and parent molecule 1c, were thus planned to enhance activity of DXR inhibitor for an herbicide application (Figure 1). 
<smiles>[R]C(=O)N(O)CCCP(=O)(O)O</smiles>
FR-900098: $R=M e, 1 b$

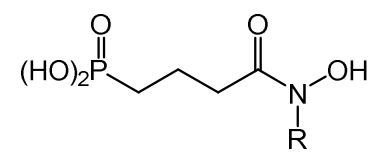

Retrohydroxamic-Fosmidomycin: $\mathrm{R}=\mathrm{H}, 1 \mathrm{c}$ Retrohydroxamic-FR-900098: $R=M e, 1 d$<smiles>[R]C(=O)N(O)CCOP(=O)=O</smiles>

Fosfoxacin: $\mathrm{R}=\mathrm{H}, 1 \mathrm{e}$ Acetyl derivative: $R=M e, 1 f$<smiles>CC(=O)[C@@H](O)[C@@H](O)COP(=O)(O)O</smiles><smiles>CC(=O)N(O)CCC(F)(F)P(=O)(O)O</smiles><smiles>O=C(CCC(F)(F)P(=O)(O)O)NO</smiles>
4

Figure 1. DXR enzyme inhibitors 1a-f referenced and the natural substrate 2. Targeted gemdifluorophosphonate $\mathbf{3}$ and retrohydroxamic-fosmidomycin $\mathbf{4}$ as $\mathbf{1 b - c}$ analogues.

\section{Results and Discussion}

It was expected that fluoro analogues of fosmidomycin, and retrohydroxamic fosmidomycin 3 and 4 would be accessible from a same key precursor, the bromodifluoromethylphosphonate 7 . Furthermore, reactions of phosphonate 7 with an appropriate alkene had to afford fluoro intermediates $\mathbf{5}$ or $\mathbf{6}$, which then would be transformed into hydroxamate by modification of the terminal alkene $\mathbf{5}$ or ester function from $\mathbf{6}$ (Scheme 1).<smiles>C=CCC(F)(F)P(=O)(O)C(=O)C=C</smiles><smiles>[3H]C(F)(F)P(=O)(Br)O[Na]</smiles><smiles>O=C(CCC(F)(F)P(=O)(O)O)NO</smiles>

Scheme 1. Retrosynthetic pathway.

In the literature, several strategies were described for the introduction of difluoromethylene group linked to a phosphonate. ${ }^{18}$ The most efficient pathway to produce quantitatively $\mathbf{7}$, has been to use the Arbusov reaction according to Davisson method, of triethyl phosphite and 
difluorodibromomethane. ${ }^{24}$ Furthermore, the transformation of bromodifluoromethylphosphonate 7 into nucleophilic Grignard or heterocuprate reagents and their reaction with allyl bromide were explored. Unfortunately, none of these reactions were successful to produce allyl difluoromethylphosphonate 5 .

Another strategy developed by $\mathrm{Hu}^{25}$ using a bimetallic redox system constituted by cobaloxime(III)/Zn and reported to initiate the radical addition of per(poly)fluoroalkyl bromides to electron deficient alkenes, has been used with success producing allyl phosphonate $\mathbf{5}^{25}$ and the phosphonobutyrate $\mathbf{6}^{25}$ in $50 \%$ and $32 \%$ yields respectively (Scheme 2). Nevertheless, as mentioned by $\mathrm{Hu}$, during the process, difluoromethylphosphonate $\mathbf{8}$ was formed as major side product, and in the case of $\mathbf{5}$, all trials to entirely remove it were unsuccessful. Furthermore, the mixture of $\mathbf{5}$ and $\mathbf{8}$ has been used for preparation of $N$-phosphonohydroxamate ester $\mathbf{1 2}$.

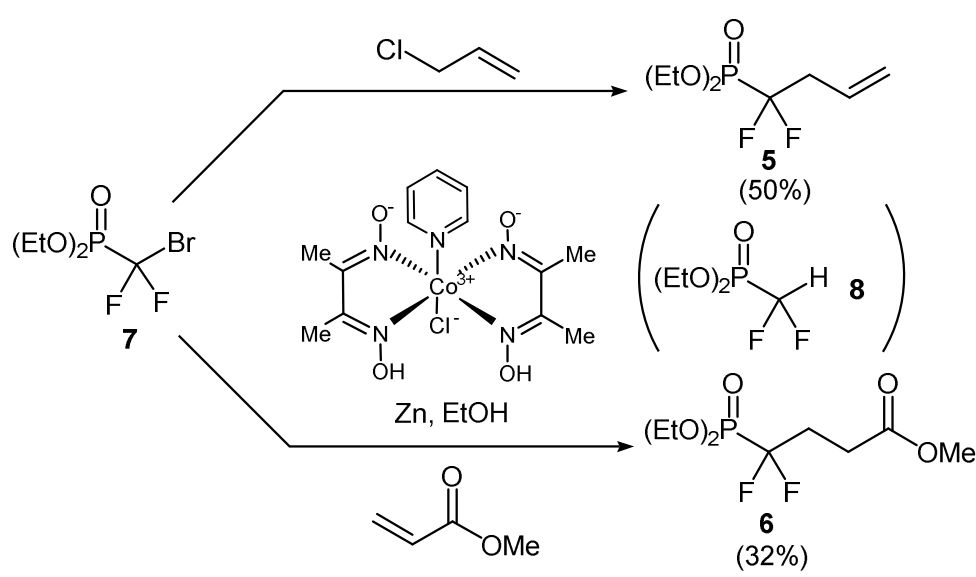

Scheme 2. Preparation of intermediates 5 and $\mathbf{6}$ by Hu's method.

The preparation of $N$-phosphonohydroxamate ester 12 was accomplished through an ozonolysis of the alkene $\mathbf{5}$ applying the Chambers's procedure. ${ }^{26}$ Due to its low stability, aldehyde $\mathbf{9}$ had to be promptly used for the next step, and its transformation by a reductive amination, using sodium cyanoborohydride in acidic conditions, ${ }^{27}$ afforded the $O$ benzylhydroxylamino phosphonate $\mathbf{1 0}$ in $68 \%$ yield. Finally, the acylation was performed in dichloromethane using acetyl chloride and triethylamine as a base. Difluoromethyl hydroxamate 11 was obtained in $86 \%$ yield. The benzyl group was cleaved using hydrogen and palladium hydroxide as catalyst, giving the difluoromethylphosphonate $\mathbf{1 2}$ analogue of FR-900098-ester (95\% yield). The hydrolysis of the phosphonate ester using trimethylsilyl bromide, ${ }^{28,29}$ followed by methanolysis at room temperature, did not afford pure phosphonic acid 3. Otherwise, another attempt by acidolysis of $\mathbf{1 2}$ and neutralization, followed by $\mathrm{N}$-acetylation also revealed not convincing for a clean access to phosphonic acid $\mathbf{3}$ (Scheme 3).

Contrary to $\mathbf{3}$, the synthesis of the difluorofosmidomycin analogue $\mathbf{4}$ has been achieved without difficulty. Firstly, the hydrolysis of the ester $\mathbf{6}$ under acidic conditions produced the corresponding carboxylic acid $\mathbf{1 3}$ in nearly quantitative yield. After chlorination and reaction 
with $O$-benzylhydroxylamine, the protected hydroxamic acid 14, was isolated in $80 \%$ yield. From compound 14, benzyl group was removed quantitatively by hydrogenolysis to give 15 . The final step of the synthesis was the deprotection of the corresponding phosphonate into phosphonic acid 4 (75\% yield), using 10 equivalents of trimethylsilyl bromide followed by a methanolysis at room temperature (Scheme 4).<smiles></smiles>

5<smiles>CCCC(F)(F)P(=O)(O)CCN(O)C(C)=O</smiles>

3

a) $\mathrm{TMSBr}, \mathrm{CH}_{2} \mathrm{Cl}_{2}$<smiles>CCO[PH](=O)C(F)(F)CC=O</smiles>

$(87 \%)$

$\mathrm{BnO}-\mathrm{NH}_{2}, \mathrm{NaBH}_{3} \mathrm{CN}$,

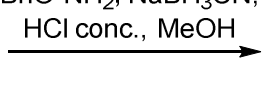

$(\mathrm{EtO})_{2}$

$\underset{\mathrm{MeC}(\mathrm{O}) \mathrm{Cl}, \mathrm{Et}}{\mathrm{CH}_{2} \mathrm{~N} \mathrm{Cl}_{2}}$<smiles>CCOP(=O)(CCCN(O)C(C)=O)C(F)(F)COO</smiles>

(95\%)<smiles>CCCON(CCC(F)(F)[PH](=O)OCC)C(C)=O</smiles>

11

$(86 \%)$

Scheme 3. Synthesis of gem-difluoro 12, analogue of FR-900098-ester.

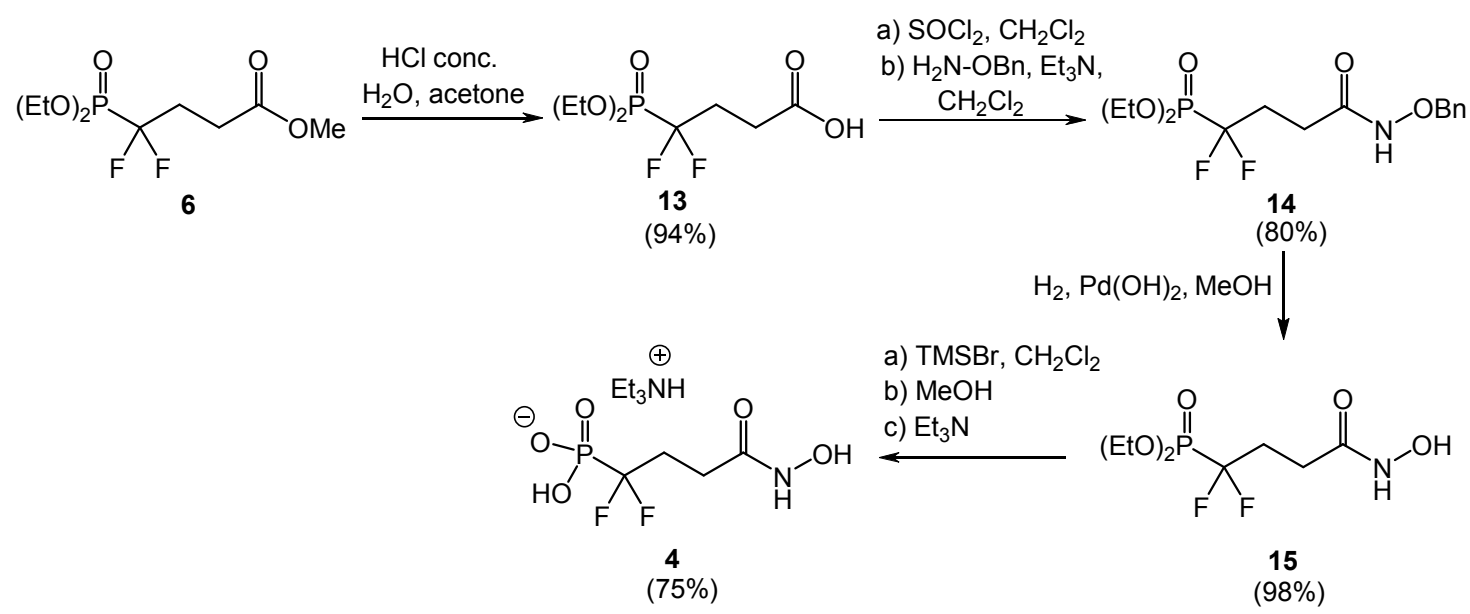

Scheme 4. Synthesis of gem-difluorinated retrohydroxamic acid triethylammonium salt 4.

\section{Conclusions}

In conclusion, using difluorobromomethylphosphonate as a key building block, we performed an efficient synthesis of new gem-difluorinated analogues of retrohydroxamic fosmidomycin and retrohydroxamic-FR-900098-ester 4 and 12. Both fluorinated fosmidomycin analogues $\mathbf{4}$ and $\mathbf{1 2}$ were not active on the biological tests for determination of herbicide activity. 


\section{Acknowledgements}

This research was supported by a Grant from Bayer CropScience and the CNRS.

\section{Experimental Section}

General. All air and/or water sensitive reactions were carried out under nitrogen atmosphere. The solvents were dried using standard methods, distilled and stored under nitrogen. Reactions were monitored by ${ }^{31} \mathrm{P}$ NMR using DMSO- $\mathrm{d}_{6}$ as internal references. Purifications by column chromatography were performed on silica gel (Merck 60 AC. 35-70 $\mu \mathrm{m}$ ).

Unless otherwise specified, NMR spectra were recorded on a BRUKER Ultra shield 400 plus instrument at $161.99 \mathrm{MHz}$ for ${ }^{31} \mathrm{P}, 376.50 \mathrm{MHz}$ for ${ }^{19} \mathrm{~F}, 400.13 \mathrm{MHz}$ for ${ }^{1} \mathrm{H}$ and $100.61 \mathrm{MHz}$ for ${ }^{13} \mathrm{C}$. The spectrometer used for low and high resolution mass spectra was electrospray ionization (ESI) WATERS Micromass Q-Tof spectrometer with as internal reference $\mathrm{H}_{3} \mathrm{PO}_{4}(0.1 \%$ in water/acetonitrile, 1:1).

The diethyl (bromodifluoromethyl)phosphonate 7, was prepared according to procedure described by Davisson. ${ }^{24}$ The diethyl 1,1-difluoro-3-butenylphosphonate $\mathbf{5}^{25,30,31}$ and methyl 4(diethoxyphosphinyl)-4,4-difluorobutyrate $6^{25}$ were synthesized using procedure reported by Hu. ${ }^{25}$ Diethyl (1,1-difluoro-3-oxopropyl)phosphonate $\mathbf{9}^{26}$ was accessible applying Chambers's procedure. $^{26}$

Diethyl (3-(benzyloxyamino)-1,1-difluoropropyl)phosphonate 10. In a $500 \mathrm{~mL}$ two-necked flask were introduced diethyl 1,1-difluoro-3-oxopropylphosphonate 9 (3.0 g, $13 \mathrm{mmol}), O$ benzyl-hydroxylamine $(1.6 \mathrm{~g}, 13 \mathrm{mmol})$ and methanol $(10 \mathrm{~mL})$. The reaction mixture was stirred for $1 \mathrm{~h}$ at room temperature. After addition of methanol $(190 \mathrm{~mL})$ the mixture was treated portionwise with sodium cyanoborohydride $(2.45 \mathrm{~g}, 39 \mathrm{mmol})$ over $30 \mathrm{~min}$. Then, aqueous hydrochloric acid $(13 \mathrm{~mL}, 37 \%)$ was added over 40 min under ice cooling. The mixture was allowed to warm up to room temperature and sodium cyanoborohydride $(0.57 \mathrm{~g}, 9.1 \mathrm{mmol})$ was added. The reaction mixture was stirred at room temperature over $2 \mathrm{~h}$. The solution was concentrated and treated with aqueous potassium hydroxide (10\%) until $p \mathrm{H}$ basic. The product was extracted with ethyl acetate $(3 \times 150 \mathrm{~mL})$, the organic layers were combined, dried over $\mathrm{MgSO}_{4}$ and concentrated under vacuum. A purification by column chromatography on silica gel with heptane/EtOAc/EtOH as eluent (100:0:0 to 60:36:4) gave $\mathbf{1 0}$ as a yellow liquid (3.0 g, $68 \%) .{ }^{31} \mathrm{P} \mathrm{NMR}\left(\mathrm{CDCl}_{3}\right): \delta 6.90(\mathrm{t}, J 107.5 \mathrm{~Hz}) .{ }^{19} \mathrm{~F} \mathrm{NMR}\left(\mathrm{CDCl}_{3}\right): \delta-111.08(\mathrm{~d}, J 107.9 \mathrm{~Hz})$. ${ }^{1} \mathrm{H}$ NMR $\left(\mathrm{CDCl}_{3}\right): \delta 1.39\left(\mathrm{t}, 6 \mathrm{H}, J 7.0 \mathrm{~Hz}, \mathrm{CH}_{3}\right), 2.30-2.46\left(\mathrm{~m}, 2 \mathrm{H}, \mathrm{CH}_{2}\right), 3.24(\mathrm{t}, 2 \mathrm{H}, J 7.2 \mathrm{~Hz}$, $\left.\mathrm{CH}_{2}\right), 4.24-4.33$ (m, 4H, $\left.\mathrm{P}(\mathrm{O}) \mathrm{OCH}_{2}\right), 4.71\left(\mathrm{~s}, 2 \mathrm{H}, \mathrm{PhCH}_{2}\right), 5.69$ (broad s, 1H, NH), 7.28-7.34 $(\mathrm{m}, 5 \mathrm{H}, \mathrm{Ph}) .{ }^{13} \mathrm{C} \mathrm{NMR}\left(\mathrm{CDCl}_{3}\right): \delta 16.4\left(\mathrm{~d}, J 5.1 \mathrm{~Hz}, \mathrm{CH}_{3}, 2 \mathrm{C}\right), 32.2(\mathrm{td}, J 20.5 \mathrm{~Hz}$ and $14.6 \mathrm{~Hz}$, $\mathrm{CH}_{2}, 1 \mathrm{C}$ ), 44.4 (q, J 5.4 Hz, $\left.\mathrm{NCH}_{2}, 1 \mathrm{C}\right), 64.5$ (d, J 6.6 Hz, P(O)OCH $\left.2,2 \mathrm{C}\right), 76.2$ (s, $\mathrm{PhCH}_{2}, 1$ C), 120.5 (td, $J 259.8 \mathrm{~Hz}$ and $215.9 \mathrm{~Hz}, \mathrm{CF}_{2}, 1 \mathrm{C}$ ), 127.9 (s, CHar, $1 \mathrm{C}$ ), 128.4 (s, CHar, $2 \mathrm{C}$ ), 
128.4 (s, CHar, 2 C), 137.7 (s, Car, 1 C). HRMS (m/z) calcd for $\mathrm{C}_{14} \mathrm{H}_{23} \mathrm{~F}_{2} \mathrm{NO}_{4} \mathrm{P}: 338.1333$. Found: 338.1327.

Diethyl (3-( $N$-(benzyloxy)acetamido)-1,1-difluoropropyl)phosphonate 11. In a $100 \mathrm{~mL}$ twonecked flask were introduced diethyl (3-(benzyloxyamino)-1,1-difluoropropyl)phosphonate $\mathbf{1 0}$ $(1.2 \mathrm{~g}, 3.6 \mathrm{mmol})$, triethylamine $(0.58 \mathrm{~mL}, 4.2 \mathrm{mmol})$ and $\mathrm{CH}_{2} \mathrm{Cl}_{2}(30 \mathrm{~mL})$. The reaction mixture was cooled to $0^{\circ} \mathrm{C}$ and acetyl chloride $(0.30 \mathrm{~mL}, 4.2 \mathrm{mmol})$ was added dropwise. The mixture was stirred overnight at room temperature. Then, water $(20 \mathrm{~mL})$ was poured on and the reaction mixture was extracted with $\mathrm{CH}_{2} \mathrm{Cl}_{2}(2 \times 50 \mathrm{~mL})$. The organic layers were combined, dried over $\mathrm{MgSO}_{4}$ and concentrated under vacuum. Purification by column chromatography on silica gel with as eluent a mixture of heptane/EtOAc/EtOH (100:0:0 to 50:45:5) gave $11(1.2 \mathrm{~g}, 86 \%) .{ }^{31} \mathrm{P}$ $\operatorname{NMR}\left(\mathrm{CDCl}_{3}\right): \delta 6.51(\mathrm{t}, J 107.0 \mathrm{~Hz}) .{ }^{19} \mathrm{~F} \mathrm{NMR}\left(\mathrm{CDCl}_{3}\right): \delta-112.34(\mathrm{~d}, J 106.7 \mathrm{~Hz}) .{ }^{1} \mathrm{H} \mathrm{NMR}$ $\left(\mathrm{CDCl}_{3}\right): \delta 1.39\left(\mathrm{t}, 6 \mathrm{H},{ }^{3} J_{\mathrm{HH}} 7.0 \mathrm{~Hz}, \mathrm{CH}_{3}\right), 2.10\left(\mathrm{~s}, 3 \mathrm{H}, \mathrm{CH}_{3}\right), 2.40-2.47\left(\mathrm{~m}, 2 \mathrm{H}, \mathrm{CH}_{2}\right), 3.93(\mathrm{t}$, $\left.2 \mathrm{H},{ }^{3} J_{\mathrm{HH}} 7.5 \mathrm{~Hz}, \mathrm{NCH}_{2}\right), 4.24-4.33\left(\mathrm{~m}, 4 \mathrm{H}, \mathrm{OCH}_{2}\right), 4.85\left(\mathrm{~s}, 2 \mathrm{H}, \mathrm{PhCH}_{2}\right), 7.35-7.43(\mathrm{~m}, 5 \mathrm{H}, \mathrm{Ph})$. ${ }^{13} \mathrm{C} \mathrm{NMR}\left(\mathrm{CDCl}_{3}\right): \delta 16.4\left(\mathrm{~d}, J 5.9 \mathrm{~Hz}, \mathrm{CH}_{3}, 2 \mathrm{C}\right), 20.6\left(\mathrm{~s}, \mathrm{CH}_{3}, 1 \mathrm{C}\right), 30.8\left(\mathrm{q}, J 18.3 \mathrm{~Hz}, \mathrm{CH}_{2}, 1\right.$ C), $38.5\left(\mathrm{~s}, \mathrm{CH}_{2}, 1 \mathrm{C}\right), 64.6\left(\mathrm{~d}, J 6.6 \mathrm{~Hz}, \mathrm{OCH}_{2}, 2 \mathrm{C}\right), 76.5\left(\mathrm{~s}, \mathrm{PhCH}_{2}, 1 \mathrm{C}\right), 119.8(\mathrm{td}, J 259.8 \mathrm{~Hz}$ and $215.9 \mathrm{~Hz}, \mathrm{CF}_{2}, 1 \mathrm{C}$ ), 128.8 (s, CHar, $2 \mathrm{C}$ ), 129.1 (s, CHar, $1 \mathrm{C}$ ), 129.3 (s, CHar, $2 \mathrm{C}$ ), 134.2 (s, Car, $1 \mathrm{C}$ ), 172.0 (s, C(O), $1 \mathrm{C}$ ). HRMS (m/z) calcd for $\mathrm{C}_{16} \mathrm{H}_{25} \mathrm{~F}_{2} \mathrm{NO}_{5} \mathrm{P}: 380.1438$. Found: 380.1448 .

Diethyl (1,1-difluoro-3-( $N$-hydroxyacetamido)propyl)phosphonate 12. In a Schlenck tube under nitrogen were introduced palladium dihydroxide on charcoal $(150 \mathrm{mg}, 10 \%)$ and $\mathbf{1 1}(1.5 \mathrm{~g}$, $4.0 \mathrm{mmol})$ in degassed ethanol $(100 \mathrm{~mL})$. The reaction mixture was stirred vigorously under a hydrogen atmosphere at room temperature for $12 \mathrm{~h}$. The reaction mixture was filtered on a celite pad and the filtrate was concentrated under vacuum to afford $\mathbf{1 2}$ as yellow liquid $(1.1 \mathrm{~g}, 95 \%)$. ${ }^{31} \mathrm{P}$ NMR $\left(\mathrm{CD}_{3} \mathrm{OD}\right): \delta 5.17(\mathrm{t}, J 108.0 \mathrm{~Hz}) .{ }^{19} \mathrm{~F} \mathrm{NMR}\left(\mathrm{CD}_{3} \mathrm{OD}\right): \delta-114.98(\mathrm{~d}, J 107.9 \mathrm{~Hz}) .{ }^{1} \mathrm{H}$ NMR (CD $3 \mathrm{OD}): \delta 1.39$ (td, $6 \mathrm{H}, J 7.1 \mathrm{~Hz}$ and $\left.0.5 \mathrm{~Hz}, \mathrm{CH}_{3}\right), 2.10\left(\mathrm{~s}, 3 \mathrm{H}, \mathrm{CH}_{3}\right), 2.35-2.45(\mathrm{~m}, 2 \mathrm{H}$, $\left.\mathrm{CH}_{2}\right), 3.87\left(\mathrm{t}, 2 \mathrm{H}, J 7.7 \mathrm{~Hz}, \mathrm{NCH}_{2}\right), 4.26-4.34\left(\mathrm{~m}, 4 \mathrm{H}, \mathrm{OCH}_{2}\right) .{ }^{13} \mathrm{C} \mathrm{NMR}\left(\mathrm{CD}_{3} \mathrm{OD}\right): \delta 14.9(\mathrm{~d}, J$ $5.1 \mathrm{~Hz}, \mathrm{CH}_{3}, 2 \mathrm{C}$ ), 18.5 (s, $\mathrm{CH}_{3}, 1 \mathrm{C}$ ), 30.3 (td, J $20.5 \mathrm{~Hz}$ and $14.6 \mathrm{~Hz}, \mathrm{CH}_{2}, 1 \mathrm{C}$ ), 40.2 (q, J 5.8 $\left.\mathrm{Hz}, \mathrm{NCH}_{2}, 1 \mathrm{C}\right), 64.6$ (d, J 7.3 Hz, $\left.\mathrm{OCH}_{2}, 2 \mathrm{C}\right), 119.4$ (td, $J 259.1 \mathrm{~Hz}$ and $218.8 \mathrm{~Hz}, \mathrm{CF}_{2}, 1 \mathrm{C}$ ), 172.2 (s, C(O), 1 C). HRMS ( $\mathrm{m} / \mathrm{z}$ ) calcd for $\mathrm{C}_{9} \mathrm{H}_{19} \mathrm{~F}_{2} \mathrm{NO}_{5} \mathrm{P}: 290.0969$, found: 290.0974 .

4-Diethoxyphosphinyl-4,4-difluorobutanoic acid 13. In a $100 \mathrm{~mL}$ two-necked flask were introduced methyl 4-(diethoxyphosphinyl)-4,4-difluorobutyrate $\mathbf{6}(4.5 \mathrm{~g}, 16.4 \mathrm{mmol})$ and acetone $(40 \mathrm{~mL})$, followed by a slow addition of hydrochloric acid solution $(22 \mathrm{~mL}, 6 \mathrm{M})$. The resulting mixture was stirred under reflux for $45 \mathrm{~min}$. The acetone was removed under vacuum and the product was extracted with diethyl ether $(3 \times 100 \mathrm{~mL})$. The organic layers were combined, dried over $\mathrm{MgSO}_{4}$ and concentrated under vacuum to give $\mathbf{1 3}$ as a colorless liquid $(4.0 \mathrm{~g}, 94 \%) .{ }^{31} \mathrm{P}$ NMR (101.25 MHz, $\left.\mathrm{CDCl}_{3}\right): \delta 6.47(\mathrm{t}, J 107.9 \mathrm{~Hz}) .{ }^{19} \mathrm{~F}$ NMR (235.33 MHz, MeOD): $\delta-114.01$ $(\mathrm{d}, J 108.2 \mathrm{~Hz}) .{ }^{1} \mathrm{H}$ NMR $\left(250 \mathrm{MHz}, \mathrm{CDCl}_{3}\right): \delta 1.38\left(\mathrm{t}, 6 \mathrm{H}, J 7.1 \mathrm{~Hz}, \mathrm{CH}_{3}\right), 2.32-2.69(\mathrm{~m}, 4 \mathrm{H}$, $\left.\mathrm{CH}_{2} \mathrm{CH}_{2}\right), 4.22-4.34\left(\mathrm{~m}, 4 \mathrm{H}, \mathrm{OCH}_{2}\right), 8.64$ (broad s, $\left.1 \mathrm{H}, \mathrm{OH}\right) .{ }^{13} \mathrm{C} \mathrm{NMR}\left(\mathrm{CDCl}_{3}\right): \delta 16.3(\mathrm{~d}, J 5.9$ $\mathrm{Hz}, \mathrm{CH}_{3}, 2 \mathrm{C}$ ), 25.8 (q, J 5.1 Hz, $\mathrm{CH}_{2}, 1 \mathrm{C}$ ), 29.2 (td, J $21.2 \mathrm{~Hz}$ and $16.0 \mathrm{~Hz}, \mathrm{CH}_{2}, 1 \mathrm{C}$ ), 64.9 (d, J 
6.6 Hz, $\mathrm{CH}_{2}, 2 \mathrm{C}$ ), 119.8 (td, J $259.8 \mathrm{~Hz}$ and $217.4 \mathrm{~Hz}, \mathrm{CF}_{2}, 1 \mathrm{C}$ ), 175.96 (s, C(O), $1 \mathrm{C}$ ). HRMS $\left(\mathrm{m} / \mathrm{z}\right.$ ) calcd for $\mathrm{C}_{8} \mathrm{H}_{16} \mathrm{~F}_{2} \mathrm{O}_{5} \mathrm{P}: 261.0703$, found: 261.0722.

Diethyl (4-(benzyloxyamino)-1,1-difluoro-4-oxobutyl)phosphonate 14. In a $250 \mathrm{~mL}$ twonecked flask were introduced 4-diethylphosphonyl-4,4-difluorobutyric acid (13, $4.0 \mathrm{~g}, 15.4$ mmol $)$ and $\mathrm{CH}_{2} \mathrm{Cl}_{2}(100 \mathrm{~mL})$. The mixture was cooled at $0{ }^{\circ} \mathrm{C}$ and thionyl chloride $(2.25 \mathrm{~mL}$, $30.8 \mathrm{mmol}$ ) was slowly added. The resulting mixture was stirred at room temperature for $4 \mathrm{~h}$, then concentrated under vacuum and evaporated twice with dry toluene $(2 \times 20 \mathrm{~mL})$. The acyl chloride was used directly without further purification. In a $250 \mathrm{~mL}$ two-necked flask were successively introduced $O$-benzyl hydroxylamine $(2.27 \mathrm{~g}, 18.4 \mathrm{mmol}), \mathrm{CH}_{2} \mathrm{Cl}_{2}(150 \mathrm{~mL})$ and triethylamine $(2.6 \mathrm{~mL}, 18.7 \mathrm{mmol})$. A solution of 4-diethoxyphosphinyl-4,4-difluorobutyric acid chloride (15.4 mmol) in $\mathrm{CH}_{2} \mathrm{Cl}_{2}(50 \mathrm{~mL})$ was added dropwise. The mixture was stirred at room temperature overnight. Then, water $(100 \mathrm{~mL})$ was poured on and the product was extracted with $\mathrm{CH}_{2} \mathrm{Cl}_{2}(3 \times 150 \mathrm{~mL})$. The organic layers were combined, dried over $\mathrm{MgSO}_{4}$ and concentrated under vacuum. The residue was purified by column chromatography on silica gel with as eluent a mixture of heptane/EtOAc/EtOH (100:0:0 to 40:54:6) affording $\mathbf{1 4}$ as yellow liquid (4.5 g, 80\%). ${ }^{31} \mathrm{P}$ NMR $\left(\mathrm{CD}_{3} \mathrm{OD}\right): \delta 5.34(\mathrm{t}, J 109.0 \mathrm{~Hz}) .{ }^{19} \mathrm{~F}$ NMR $\left(\mathrm{CD}_{3} \mathrm{OD}\right): \delta-115.66(\mathrm{~d}, J 109.2 \mathrm{~Hz}) .{ }^{1} \mathrm{H}$ NMR (CD $\left.{ }_{3} \mathrm{OD}\right): \delta 1.38\left(\mathrm{t}, 6 \mathrm{H}, J 7.1 \mathrm{~Hz}, \mathrm{CH}_{3}\right), 2.30-2.50\left(\mathrm{~m}, 4 \mathrm{H}, \mathrm{CH}_{2} \mathrm{CH}_{2}\right), 4.24-4.34(\mathrm{~m}, 4 \mathrm{H}$, $\left.\mathrm{OCH}_{2}\right), 4.84\left(\mathrm{~s}, 2 \mathrm{H}, \mathrm{PhCH}_{2}\right), 7.35-7.45(\mathrm{~m}, 5 \mathrm{H}, \mathrm{Ph}) .{ }^{13} \mathrm{C} \mathrm{NMR}\left(\mathrm{CDCl}_{3}\right): \delta 16.4(\mathrm{~d}, J 5.1 \mathrm{~Hz}$, $\mathrm{CH}_{3}, 2 \mathrm{C}$ ), 24.9 (s, $\mathrm{CH}_{2}, 1 \mathrm{C}$ ), 29.7 (q, J $19.0 \mathrm{~Hz}, \mathrm{CH}_{2}, 1 \mathrm{C}$ ), 64.7 (s, $\mathrm{OCH}_{2}, 2 \mathrm{C}$ ), 78.1 (s, $\mathrm{PhCH}_{2}, 1 \mathrm{C}$ ), 120.1 (td, J $259.8 \mathrm{~Hz}$ and $215.9 \mathrm{~Hz}, \mathrm{CF}_{2}, 1 \mathrm{C}$ ), 128.5 (s, CHar, $2 \mathrm{C}$ ), 128.6 (s, CHar, 1 C), 129.1 (s, CHar, 2 C), 135.4 (s, Car, 1 C), 168.9 (s, C(O), 1 C). HRMS ( $m / z$ ) calcd for $\mathrm{C}_{15} \mathrm{H}_{23} \mathrm{~F}_{2} \mathrm{NO}_{5} \mathrm{P}: 366.1282$. Found: 366.1281 .

Diethyl (1,1-difluoro-4-(hydroxyamino)-4-oxobutyl)phosphonate 15. Proceeding as for 12, compound 15 was obtained from 14 as yellow liquid $(1.1 \mathrm{~g}, 98 \%) .{ }^{31} \mathrm{P} \mathrm{NMR}\left(\mathrm{CDCl}_{3}\right): \delta 6.51(\mathrm{t}, \mathrm{J}$ $108.0 \mathrm{~Hz}) .{ }^{19} \mathrm{~F}$ NMR $\left(\mathrm{CDCl}_{3}\right): \delta-111.86(\mathrm{~d}, \mathrm{~J} 107.9 \mathrm{~Hz}) .{ }^{1} \mathrm{H}$ NMR $\left(\mathrm{CDCl}_{3}\right): \delta 1.31(\mathrm{t}, 6 \mathrm{H}, \mathrm{J} 7.0$ $\left.\mathrm{Hz}, \mathrm{CH}_{3}\right), 2.2-2.5\left(\mathrm{~m}, 4 \mathrm{H}, \mathrm{CH}_{2}-\mathrm{CH}_{2}\right), 4.15-4.25\left(\mathrm{~m}, 4 \mathrm{H}, \mathrm{OCH}_{2}\right), 8.70$ (broad s, 0.8H), 9.87 (broad s, 0.8H). ${ }^{13} \mathrm{C} \mathrm{NMR}\left(\mathrm{CDCl}_{3}\right): \delta 16.3\left(\mathrm{~d}, J 5.1 \mathrm{~Hz}, \mathrm{CH}_{3}, 2 \mathrm{C}\right), 24.5\left(\mathrm{CH}_{2}, 1 \mathrm{C}\right), 29.7$ (q, $J$ $\left.19.3 \mathrm{~Hz}, \mathrm{CH}_{2}, 1 \mathrm{C}\right), 65.0\left(\mathrm{~d}, J 6.6 \mathrm{~Hz}, \mathrm{OCH}_{2}, 2 \mathrm{C}\right), 120.1\left(\mathrm{td}, J 259.8 \mathrm{~Hz}\right.$ and $216.6 \mathrm{~Hz}, \mathrm{CF}_{2}, 1$ C), $169.4(\mathrm{~s}, \mathrm{C}(\mathrm{O}), 1 \mathrm{C})$. HRMS ( $\mathrm{m} / \mathrm{z}$ ) calcd for $\mathrm{C}_{8} \mathrm{H}_{17} \mathrm{~F}_{2} \mathrm{NO}_{5} \mathrm{P}: 276.0812$. Found: 276.0809.

Triethylammonium (1,1-difluoro-4-(hydroxyamino)-4-oxobutyl)phosphonate 4. In a $50 \mathrm{~mL}$ three-necked flask were introduced $15(1 \mathrm{mmol})$ and $\mathrm{CH}_{2} \mathrm{Cl}_{2}(10 \mathrm{~mL})$. The mixture was stirred in an ice bath $\left(\mathrm{T}=0^{\circ} \mathrm{C}\right)$ and trimethylsilylbromide $(1.3 \mathrm{~mL}, 10 \mathrm{mmol})$ was added. The reaction mixture was stirred at room temperature for $14 \mathrm{~h}$ and concentrated to dryness under vacuum. Methanol $(10 \mathrm{~mL})$ was added and the resulting mixture was stirred at room temperature for $2 \mathrm{~h}$ and then concentrated under vacuum. The crude was dissolved in methanol $(10 \mathrm{~mL})$ and triethylamine $(1.4 \mathrm{~mL}, 10 \mathrm{mmol})$ was added slowly. The solvent was removed under vacuum. The crude was dissolved in water $(50 \mathrm{~mL})$ and the suspension filtered off. The aqueous layer was washed with chloroform $(3 \times 50 \mathrm{~mL})$. After concentration under vacuum, the salt 4 was obtained as a colorless gum $(240 \mathrm{mg}, 75 \%) .{ }^{31} \mathrm{P}$ NMR $\left(\mathrm{D}_{2} \mathrm{O}\right): \delta 4.75(\mathrm{t}, J 95.1 \mathrm{~Hz}) ;{ }^{19} \mathrm{~F}$ NMR $\left(\mathrm{D}_{2} \mathrm{O}\right): \delta-$ $113.50(\mathrm{~d}, J 95.2 \mathrm{~Hz}) .{ }^{1} \mathrm{H}$ NMR $\left(\mathrm{D}_{2} \mathrm{O}\right): \delta 1.14\left(\mathrm{t}, 9 \mathrm{H}, J 7.3 \mathrm{~Hz}, \mathrm{CH}_{3}\right), 2.1-2.3\left(\mathrm{~m}, 2 \mathrm{H}, \mathrm{CH}_{2}\right), 2.46$ 
(t, 2H, J $\left.7.3 \mathrm{~Hz}, \mathrm{CH}_{2} \mathrm{C}(\mathrm{O})\right), 3.06\left(\mathrm{q}, J 7.3 \mathrm{~Hz}, 6 \mathrm{H},{ }^{5} \mathrm{CH}_{2}\right) .{ }^{13} \mathrm{C} \mathrm{NMR}\left(\mathrm{D}_{2} \mathrm{O}\right): \delta 8.2\left(\mathrm{~s}, \mathrm{CH}_{3}, 3 \mathrm{C}\right)$, 27.1 (q, J $\left.5.4 \mathrm{~Hz}, \mathrm{CH}_{2} \mathrm{C}(\mathrm{O}), 1 \mathrm{C}\right), 29.4\left(\mathrm{td}, J 21.2 \mathrm{~Hz}\right.$, and $\left.14.6 \mathrm{~Hz}, \mathrm{CH}_{2}, 1 \mathrm{C}\right), 46.6\left(\mathrm{~s}, \mathrm{NCH}_{2}, 3\right.$ C), 122.0 (td, J $257.6 \mathrm{~Hz}$ and $\left.196.9 \mathrm{~Hz}, \mathrm{CF}_{2}, 1 \mathrm{C}\right), 178.8$ (s, C(O), $\left.1 \mathrm{C}\right)$. HRMS (m/z) calcd for $\mathrm{C}_{4} \mathrm{H}_{9} \mathrm{~F}_{2} \mathrm{NO}_{5} \mathrm{P}: 220.0186$. Found 220.0191 .

\section{References}

1. Kuzuyama T.; Takahashi S.; Seto H. Biosci., Biotechnol., Biochem. 1999, 63, 776. http://dx.doi.org/10.1271/bbb.63.776

2. Cornish, R. M.; Roth J. R.; Poulter C. D. J. Bacteriol. 2006, 188, 1444. http://dx.doi.org/10.1128/JB.188.4.1444-1450.2006

3. Brown A.; Parish T. BMC Microbiol. 2008, 8, 78. http://dx.doi.org/10.1186/1471-2180-8-78

4. Odom A. R.; Van Voorhis, W. C. Mol. Biochem. Parasitol. 2010, 170, 1547. http://dx.doi.org/10.1016/J.molbiopara.2009.12.001

5. Zeidler, J.; Schwender, J.; Müller, C.; Wiesnerb, J.; Weidemeyer, C.; Beck, E.; Jomaa, H.; Lichtenthaler H. K. Z. Naturforsch., C: J. Biosci. 1998, 53, 980.

6. Fellermeier, M.; Kis, K.; Sagner, S.; Maier, U.; Bacher, A.; Zenk M. H. Tetrahedron Lett. 1999, 40, 2743. http://dx.doi.org/10.1016/S0040-4039(99)00361-5

7. Kuzuyama, T.; Shimizu T.; Takahashi S.; Seto H. Tetrahedron Lett. 1998, 39, 7913. http://dx.doi.org/10.1016/S0040-4039(98)01755-9

8. Jomaa H.; Wiesner J.; Sanderbrand S.; Altincicek B.; Weidemeyer C.; Hintz M.; Türbachova I.; Eberl M.; Zeidler J.; Lichtenthaler H. K.; Soldati D.; Beck E. Science 1999, 285, 1573. http://dx.doi.org/10.1126/science.285.5433.1573

9. Haemers, T.; Wiesner, J.; Van Poecke, S.; Goeman, J.; Henschker, D.; Beck, E.; Jomaa, H.; Van Calenbergh S. Bioorg. Med. Chem. Lett. 2006, 16, 1888. http://dx.doi.org/10.1016/J.bmcl.2005.12.082

10. Kuntz L.; Tritsch D.; Grosdemange-Billiard C.; Hemmerlin A.; Willem A.; Bach T. J.; Rohmer M. Biochem. J. 2005, 386, 127. http://dx.doi.org/10.1016/J.bmcl.2005.12.082

11. Haemers T.; Wiesner J.; Gießmann D.; Verbrugghen T.; Hillaert U.; Ortmann R.; Jomaa H.; Link A.; Schlitzer M; Van Calenbergh S. Bioorg. Med. Chem. 2008, 16, 3361. http://dx.doi.org/10.1016/J.bmc.2007.12.001

12. Woo Y.-H.; Fernandes R. P. M.; Proteau P. J. Bioorg. Med. Chem. 2006, 14, 2375. http://dx.doi.org/10.1016/J.bmc.2005.11.012

13. Borrmann S.; Adegnika A. A.; Matsiegui P.-B.; Issifou S.; Schindler A.; Mawili-Mboumba D. P.; Baranek T.; Wiesner J.; Jomaa H.; Kremsner P. G. J. Infect. Dis. 2004, 189, 901. http://dx.doi.org/10.1086/381785 
14. Borrmann S.; Lundgren I.; Oyakhirome S.; Impouma B.; Matsiegui P.-B.; Adegnika A. A.; Issifou S.; Kun J. F. J.; Hutchinson D.; Wiesner J.; Jomaa H.; Kremsner P. G. Antimicrob. Agents Chemother. 2006, 50, 2713. http://dx.doi.org/10.1128/AAC.00392-06

15. Ruangweerayut R.; Looareesuwan S.; Hutchinson D.; Chauemung A.; Banmairuroi V.; NaBangchang K. Malar. J. 2008, 7, 225. http://dx.doi.org/10.1186/1475-2875-7-225

16. Kamuro, Y.; Kawai, T.; Kakiushi T. FuJisawa Pharmaceutical Co. Ltd, Eur. Pat. 0256785, 1988; Chem. Abstr. 1988, 109, 18860.

17. Verbrugghen, T.; Cos, P.; Maes, L.; Van Calenbergh S. J. Med. Chem. 2010, 53, 5342. http://dx.doi.org/10.1021/Jm100211e

18. Romanenko, V. D.; Kukhar V. P. Chem. Rev. 2006, 106, 3868. http://dx.doi.org/10.1021/cr051000q

19. Blackburn, G. M.; Kent, D. E.; Koklmann F. J. Chem. Soc. Perkin Trans 1 1984, 1119. http://dx.doi.org/10.1039/p19840001119

20. Blackburn, G. M.; England, D. A.; Kolkmann F. J. Chem. Soc. Chem. Commun. 1981, 17, 930. http://dx.doi.org/10.1039/c39810000930

21. Chambers, R. D.; O’Hagan, D.; Lamont, R. B.; Jaina S. C. J. Chem. Soc. Chem. Comm. 1990, 15, 1053. http://dx.doi.org/10.1039/c39900001053

22. Otaka, A.; Mitsuyama E.; Watanabe J.; FuJi N. Biopolymers 2004, 76, 140. http://dx.doi.org/10.1002/bip.10570

23. Merino P. Chemical Synthesis of Nucleoside Analogues; Ed.; John Wiley and Sons, 2013, 224.

24. Davisson, V. J.; Woodside, A. B.; Neal, T. R.; Stremler, K. E.; Muehlbacher, M.; Poulter C. D. J. Org. Chem. 1986, 51, 4768. http://dx.doi.org/10.1021/Jo00375a005

25. Hu, C.-M.; Chen J. J. Chem. Soc. Perkin Trans. 1 1993, 327. http://dx.doi.org/10.1039/p19930000327

26. Chambers, R. D.; Jaouhari, R.; O’Hagan D. J. Fluorine Chem. 1989, 44, 275. http://dx.doi.org/10.1016/S0022-1139(00)83944-5

27. Schlüter, K.; Walter, R. D.; Bergmann, B.; Kurz T. Eur. J. Med. Chem. 2006, 41, 1385. http://dx.doi.org/10.1016/J.eJmech.2006.06.015

28. Allen, M. C.; Fuhrer, W.; Tuck, B.; Wade, R.; Wood J. M. J. Med. Chem. 1989, 32, 1652. http://dx.doi.org/10.1021/Jm00127a041

29. Shahgaldian, P.; Coleman, A. W.; Kalchenko V. I. Tetrahedron Lett. 2001, 42, 577. http://dx.doi.org/10.1016/S0040-4039(00)02003-7

30. Burton, D. J.; Takei, R.; Shin-Ya S. J. Fluorine Chem. 1981, 18, 197. http://dx.doi.org/10.1016/S0022-1139(00)82316-7

31. Burton, D. J.; Sprague L. G. J. Org. Chem. 1989, 54, 613. http://dx.doi.org/10.1021/Jo00264a021 